\title{
A Rare Presentation of Endometriosis with Recurrent Massive Hemorrhagic Ascites which Can Mislead
}

\author{
Fidia Mumtahana ${ }^{1}$, Jun Jiao ${ }^{1}$, Baoxia Cui ${ }^{1 *}$
}

\section{Article History:}

Received September 2013

Accepted October 2013

Available online January 2014

Keywords:

Endometriosis

Hemorrhagic Ascites

Corresponding Author:

Baoxia Cui , Department of

Gynecology, Qilu Hospital of

Shandong University, Jinan,

Shandong Province, China.

Tel: +8613791122632

Email: cuibaoxia@sdu.edu.cn

\begin{abstract}
:
Endometriosis is an estrogen dependent disease where active endometrial tissue is present in extra uterine sites. Presence of recurrent massive hemorrhagic ascites due to endometriosis is a rare phenomenon which can mislead physicians to other pathological conditions like malignancy and wrong treatment protocol may be applied. For confirming the cause of this huge bloody ascites laparoscopy or laparotomy followed by biopsy is significantly essential.
\end{abstract}

1. Department of Gynecology, Qilu Hospital of Shandong University, Jinan, Shandong Province, China. 


\section{Introduction:}

Massive hemorrhagic ascites secondary to endometriosis is rare (1) and recurrent hemorrhagic ascites due to endometriosis is hardly seen in clinic. Huge hemorrhagic ascites in reproductive age may be a complication of endometriosis but proper diagnosis is essential for excluding malignancy.

\section{Case Report:}

A 36 years nullipara Chinese female patient was admitted in the gynecological department of Qilu Hospital of Shandong University with the diagnosis of endometriosis and presentation of huge ascites due to unknown cause. She had abdominal distention for one month and abdominal pain especially after food intake. She also had dysmenorrhea for more than 10 years and infertility for 5 years. Dysmenorrhea was progressively increasing with menstrual volume and clotted blood.

On examination the patient had a normal body built with a distended abdomen. No other abnormalities were found except for positive shifting dullness which is consistent with ascites. On gynaecological examination uterus was found to be 8 weeks pregnancy size, hard and non-tender with normal both sided adnexa. From her blood biochemistry she was found mild anemic with elevated Cancer Antigen-125 (CA-125: 78.23 U/L) and other tests were normal. Her ultrasound and CT scan revealed small fibroid in the posterior abdominal wall with a large number of free fluids in the abdominal cavity; she had an abdominal paracentesis which obtained $3000 \mathrm{ml}$ of hemorrhagic ascitic fluid. Analysis of ascitic fluid showed signs of inflammation and high count of erythrocyte $(++++)$ but there was no acidfast bacilli or cancer cells. Her endoscopy and colonoscopy examination were normal. Her anti-TB antibody showed weakly positive along with weakly positive Purified Protein Derivative test (PPD) so a provisional diagnosis of tuberculous peritonitis was made. Medication given to her included anti tubercular drugs and steroids. Her condition improved and she was discharged with anti-TB drug regime.
After 10 days of discharge, distention of her abdomen again gradually returned along with abdominal pain. Her dysmenorrhea remained same with excessive bleeding, painful micturation and defecation mostly during menstruation. After 5 months she was again admitted in the gynecology department with the same distended abdomen. She was found severe anemic and except shifting dullness no abnormality was found. From her gynecological examination her uterus was found to be 8 weeks pregnancy size with normal adnexa. Her blood hemoglobin was - 78 g/L, CA-125: 86.6 $\mathrm{U} / \mathrm{L}$, test for tuberculosis and other tests were negative. Her X-ray, ECG, colonoscopy were normal like before. Her ultrasound showed- large number of free fluid in the abdomen which is about $7.2 \mathrm{~cm}$ deep and a small fibroid in the posterior wall of the uterus. Left sided ovary was normal but in the right sided ovary a $1.9 \times 1.6 \mathrm{~cm}$ mass was found but the nature of the mass could not be revealed. Doppler study of the mass showed circular blood flow which was inconclusive. Her D\&C report showed proliferative advanced endometriosis. Ascitic fluid analysis from abdominal paracentesis (2500 $\mathrm{ml}$ of fluid) showed presence of inflammation, blood and high level of tumor markers (CA 125: >5009U/L, CA 19-9: 694.5 U/L).

As the cause of recurrent huge ascites and nature of the ovarian mass was unknown laparoscopic examination was planned but due to severe adhesion in the abdominal cavity laparoscopy was not possible. Exploratory laparoscopy was done with the consent of the family. Fibrosed and scar tissue were visible in different parts of the peritoneum and bowel with dense adhesion. After blunt separation of adhesion the uterus was found about 8 weeks pregnancy size attached with surrounding structures. A small ill-defined fibroid was present in the posterior wall and two ovaries were packed within fibrosed scar tissues. In the left ovary about $2 \times 2 \mathrm{~cm}$ cyst was found which was not seen in the ultrasound. In the right ovary a cystic mass was found which was about $3 \times 2 \mathrm{~cm}$. Violet-blue nodular lesions were found in the pouch of Douglas and rectal wall. Peritoneal adhesion, bilateral ovarian 
cyst, uterine fibroid, endometrial tissue, nodules in the pouch of Douglas and rectum were removed. They were send for a quick pathological test which showed- left ovarian endometrioma and right ovarian corpus luteal cyst with peritoneal endometriosis, but there was no trace of tuberculosis or any abnormal growth. Upper abdomen was examined but no palpable lesion was found. For fertility requirement no more extended surgery was done and abdomen was closed. Goserelin acetate (GnRH agonist) injection was prescribed on a monthly basis for a certain period for severe endometriosis. Postoperative recovery was good and no more recurrence of ascites was reported.

\section{Discussion:}

Endometriosis is a benign estrogen dependent gynaecological disease where functioning endometrium is found in extra uterine sites of the body usually in ovaries, uterus, fallopian tubes, ovarian ligaments, pouch of Douglas, bowel, rectum, urinary tract, chest cavity, skin, surgical scars etc (23 ). The appearance of endometriotic lesion vary from a few minimal lesions to large ovarian endometriotic cyst (endometrioma) filled with old chocolate color blood. Usually in the ovaries it distorts tubo-ovarian anatomy and in severe condition causes extensive pelvic adhesion with distortion of the pelvic anatomy which results in pain and infertility (3-4).

The prevalence is mostly in reproductive age where the percentage is $6-10 \%$ in females. But prevalence is difficult to determine as some times the patient remains asymptomatic or have diverse symptoms (2, 4). The usual manifestation includes dysmenorrhea, menstrual disturbance, infertility, pelvic pain, gastrointestinal symptoms, urogenital symptoms, migraine, fibromyalgia etc $(2,4)$. However abdominal distention due to huge ascites secondary to endometriosis is an uncommon presentation and recurrent massive hemorrhagic ascites is extremely rare which misleads to ovarian cancer. Abdominal pain may arise from rupture of endometrioma and consecutive irritation of serosal surfaces by free blood causing ascites and adhesions. The fluid is usually exudative (1).
The diagnosis of endometriosis needs long time as the symptoms are confusing with other conditions and lack specific diagnostic tool. Ultrasound may help in detection of endometrioma but it is not able to detect the diffuse small lesions (4). Other imaging modules are expensive and the results may not be very satisfactory. It is reported that tumor marker CA 125 is higher in endometriosis but CA 125 is also higher in ovarian cancer, tubercular peritonitis, uterine fibroid, PID and other diseases. Again huge ascites with adnexal mass and raised CA 125 primarily give the impression of ovarian malignancy (5). Another tumor marker CA 19-9 is elevated in pancreatic, colonic and ovarian cancer. But high level of CA 19-9 is also demonstrated in benign conditions like benign hepatobiliary lesions, benign lung diseases, DM, ovarian cyst, endometriosis and pregnancy (6). Only surgical intervention by laparoscopy or laparotomy is considered as "gold standard" for diagnosis of endometriosis (4).

We refer ascitic fluid as hemorrhagic ascites when it contains more than 50,000 $\mathrm{RBC} /$ cubic $\mathrm{mm}$ and the main cause is ovarian cancer, tuberculous peritonitis and hepatoma (1). In the prior of this case patient was found anemic with elevated CA125 but the imaging reports showed fibroid and normal adnexa without any mass but with huge ascites which contained high count of RBC however no cancer cells or acid-fast bacilli. Though her blood anti-TB antibody and PPD test were weakly positive and other tests were normal, she was diagnosed to have endometriosis with tuberculous peritonitis as tuberculosis is difficult to diagnose as often the results of diagnosis come negative or inaccurate (5) and it was considered the only explanation for the massive hemorrhagic ascites.

The main objective of the treatment of endometriosis is pain relief and correction of infertility. Now it is known that estrogen stimulates the growth of this ectopic endometrial tissue. In patient with endometriosis estrogen production is higher and the sources of which are: ovary, conversion of androgens to estrogen in adipose tissue and local endometriotic tissue. To suppress this estrogen level 
Androgens, GnRH agonists, Progestagens and Combined oral contraceptives are used (3). Surgical options include laparoscopic excision with ablation of the lesions and adhesions along with laparoscopic cystectomy and hysterectomy with or without bilateral oophorectomy (7).

\section{Conclusion :}

Recurrent huge ascites secondary to endometriosis is very rare. However, this condition is important as massive hemorrhagic ascites in reproductive age can be mistaken for malignancy and other diseases and the chance of getting mistreatment is high which can be dangerous. If dysmenorrhea, pelvic pain, abdominal distension are present with paracentesis that reveals RBC but no evidence of malignant cells then laparoscopy or laparotomy and biopsy may be still necessary for excluding malignancy.

\section{Conflict of interest statement:}

We declare that we have no conflict of interest.

\section{Figures :}

Figure1. Laparoscopic view showing extensive adhesion with fibroids in the posterior wall of uterus, packed ovaries and visible hemorrhagic ascites.

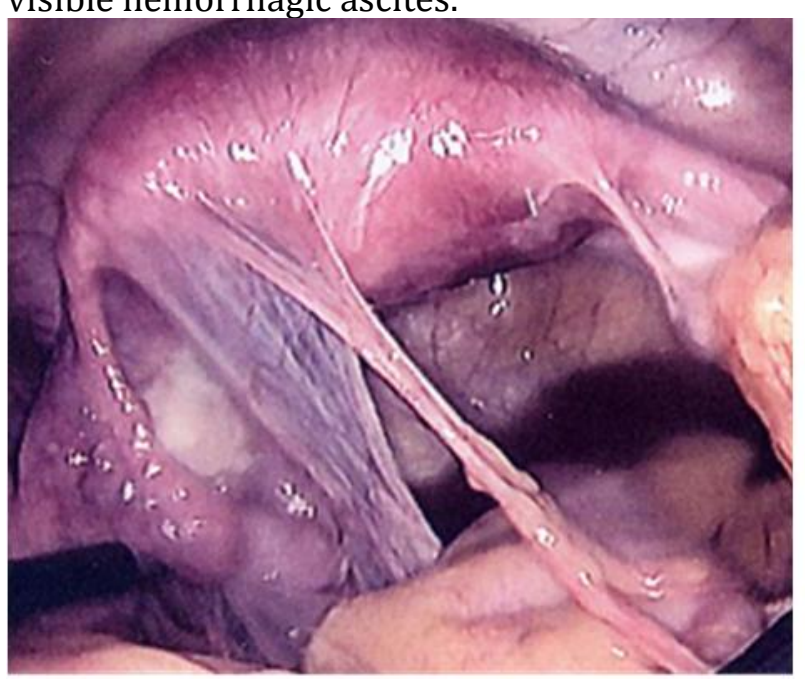

Figure2. Endometriotic lesions in the pouch of douglas

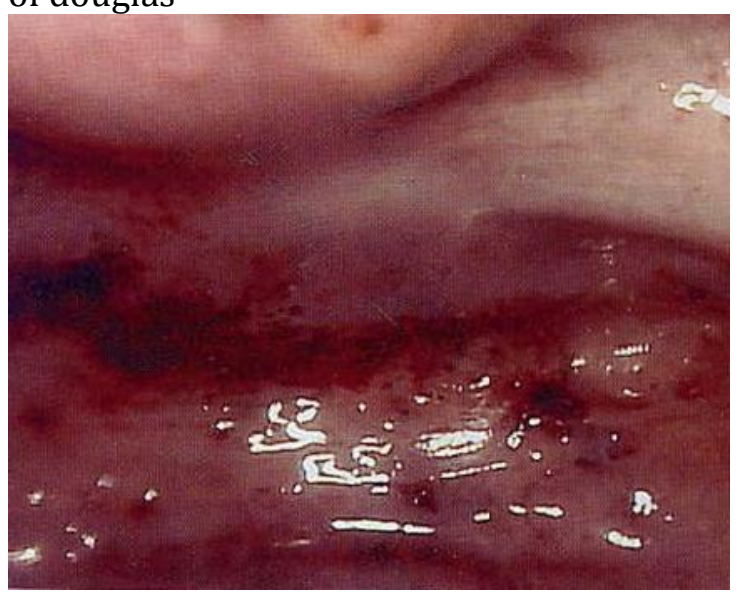




\section{References:}

1. Lodha, A., Klein, T., Elish., \& Tarkovsky, R. Endometriosis: A Rare Presentation as Hemorrhagic Ascites. Practical Gastroenterology, 2008; 32(10):48-9.

2. Cirstoiu, M., Bodean, O., Secara. D., Munteanu, O., \& Cirstoiu, C. Case study of a rare form of endometriosis. Journal of Medicine and Life. 2013; 6(1):68-71.

3. Cristescu, C., Velişcu, A., Marinescu, B., Pătraşcu, A., Traşcă, E.T., \& Pop, O.T . Endometriosis - clinical approach based on histological findings. Romanian Journal of Morphology \& Embryology. 2013; 54(1):91-7.

4. Giudice, L. C., \& Kao, L. C. Endometriosis. Lancet. 2004; 364(9447):1789-99.

5. Sheth, S. S. (1996). Elevated CA 125 in advanced abdominal or pelvic tuberculosis. International Journal of Gynecology \& Obstetrics. 1996; 52(2): 167-71.

6. Kaya, C., Cengiz, H., Ekin, M., \& Yasar, L.Transverse vaginal septum: a benign reason for elevated serum CA 19-9 and CA 125 levels. Archives of Gynecology and Obstetrics. 2012; 286(3):821-3.

7. Golfier, F., \& Sabra, M. Surgical management of endometriosis. Journal de Gynecologie Obstetrique et Biologie de la Reproduction. 2007; 36(2):162-72. 